\title{
An Adaptive Data Collection Procedure for Call Prioritization
}

\author{
Jean-Francois Beaumont ${ }^{1}$, Cynthia Bocci ${ }^{2}$, and David Haziza ${ }^{3}$
}

\begin{abstract}
We propose an adaptive data collection procedure for call prioritization in the context of computer-assisted telephone interview surveys. Our procedure is adaptive in the sense that the effort assigned to a sample unit may vary from one unit to another and may also vary during data collection. The goal of an adaptive procedure is usually to increase quality for a given cost or, alternatively, to reduce cost for a given quality. The quality criterion often considered in the literature is the nonresponse bias of an estimator that is not adjusted for nonresponse. Although the reduction of the nonresponse bias is a desirable goal, we argue that it is not a useful criterion to use at the data collection stage of a survey because the bias that can be removed at this stage through an adaptive collection procedure can also be removed at the estimation stage through appropriate nonresponse weight adjustments. Instead, we develop a procedure of call prioritization that, given the selected sample, attempts to minimize the conditional variance of a nonresponse-adjusted estimator subject to an overall budget constraint. We evaluate the performance of our procedure in a simulation study.
\end{abstract}

Key words: Adaptive collection design; nonresponse bias; nonresponse variance; nonresponse weight adjustment; paradata; responsive collection design.

\section{Introduction}

The focus of this article is the prioritization of calls in the context of Computer-Assisted Telephone Interview (CATI) surveys. We develop an adaptive data collection procedure that attempts to maximize quality given a certain budget. Our procedure is adaptive in the sense that the effort assigned to a sample unit may vary from one unit to another and may also vary during data collection, which requires the use of paradata. Paradata are data about the data collection process, such as response rates by subgroups of the sample at different time points of data collection.

The quality criterion often considered in the literature is the nonresponse bias of an estimator that is not adjusted for nonresponse. Although the reduction of the nonresponse bias is a desirable goal, we believe that it is not a useful criterion to apply at the data

1 Statistics Canada, 100 Tunney's Pasture Driveway, R.H. Coats Bldg., 16-B Ottawa K1A 0T6, Canada. Email: Francois.Beaumont@statcan.gc.ca

2 Statistics Canada, 100 Tunney's Pasture Driveway, R.H. Coats Bldg., 18-E Ottawa K1A 0T6, Canada. Email: Cynthia.Bocci@statcan.gc.ca

3 Université de Montréal, Département de mathématiques et de statistique, Pavillon André Aisenstadt, Case postale 6128, Montréal H3C 3J7, Canada. Email: david.haziza@umontreal.ca

Acknowledgments: The authors are indebted to Joël Bissonnette of Statistics Canada for his programming of the call prioritization procedure used in the simulations. They would also like to thank all the reviewers for their comments, which greatly improved the overall quality of the article. 
collection stage of a survey because the bias that can be removed at this stage through an adaptive collection procedure can also be removed at the estimation stage through appropriate nonresponse weight adjustments. For instance, we could consider a collection procedure that prioritizes cases to be interviewed so as to equalize response rates between domains of interest and then use an estimator that is not adjusted for nonresponse. In terms of nonresponse bias, we expect this strategy to be equivalent to using an estimator that adjusts design weights by the inverse of response rates within domains of interest along with a data collection procedure where cases are selected at random. The reasoning is that auxiliary information known for both respondents and nonrespondents is necessary to reduce the nonresponse bias. (Note that the auxiliary information is the domain information in the above example.) Whether this information is used at the data collection stage or not should not make a difference in terms of nonresponse bias as long as it is used at the estimation stage. This is confirmed in our empirical study (see Section 4). Therefore, the quality criterion that we suggest to minimize is the variance of a nonresponse-adjusted estimator conditional on the selected sample. We use the term nonresponse variance for this conditional variance as it emerges only because of nonresponse and has nothing to do with sampling. This variance disappears in the absence of nonresponse.

Before describing our call prioritization procedure in Section 3, we first provide a selected literature review in Section 2. Section 4 presents the results of a simulation study that evaluates the properties of our procedure and a few alternatives. The conclusion is given in Section 5, which includes some suggestions for potential improvement.

\section{Literature Review}

The literature on adaptive collection designs, sometimes called adaptive survey designs, responsive collection designs, responsive survey designs or simply responsive designs, is fairly recent. In our context, we prefer the terms adaptive collection designs and responsive collection designs as they make it clear that we are concerned with improvements in data collection methods so that any confusion with the different notion of adaptive sampling designs, which are typically used to sample from rare populations, is avoided.

Groves and Heeringa (2006) defined a responsive survey design as one that uses paradata to guide changes in the features of data collection in order to achieve higher quality estimates per unit cost. Three examples of features of data collection are the data collection mode, the use of incentives and the call prioritization procedure. The implementation of responsive designs in practice requires defining what is meant by quality and determining suitable quality indicators. A cost function must also be chosen. There are two other main concepts underlying the framework of Groves and Heeringa (2006): phase and phase capacity. A phase is a period of data collection during which the same set of methods is used. The first phase is used to gather information about data collection features. In subsequent phases, features are modified (e.g., subsampling of nonrespondents, larger incentives, etc.). A given phase is continued until it reaches its phase capacity, which is typically judged by the stability of some indicator (e.g., an estimate) as the phase matures. Axinn et al. (2011) recently evaluated the consequences of implementing responsive design methods. They studied the extent to which a responsive design altered conclusions reached from analyses of multivariate models by comparing 
differences between model coefficients obtained using the main phase sample to those obtained from the responsive phase sample. They concluded that the addition of a responsive design phase can add very different people to the respondent pool, creating significant differences in the magnitude of model coefficients.

Schouten et al. (2009) proposed an indicator of nonresponse bias, called R-indicator, as an alternative to response rates. An R-indicator is sometimes chosen as the quality indicator to be used in conjunction with an adaptive collection design. The proposed $\mathrm{R}$-indicator is a function of estimated probabilities of response to the survey and is designed to measure the representativeness of the respondents to the complete sample. It is constructed using the variability of the response probabilities. A large value of the R-indicator is associated with a low variability of the response probabilities. One drawback of this indicator is that it depends on the proper choice of a nonresponse model; that is, a model for the indicators of response to the survey. In particular, the R-indicator depends on the proper choice of explanatory variables used to model the response probabilities. For instance, if no explanatory variable is included in the nonresponse model, the R-indicator is equal to 1 , which is the best value it can reach. Thus, a poor choice of explanatory variables may lead to an artificially large value of the indicator yet does not divulge anything about the actual nonresponse bias. Indeed, the nonresponse bias may vary from one variable of interest to another. Since the R-indicator is independent of any of these variables, it can only provide limited information about nonresponse bias. The authors also considered the maximal bias of an estimator that is not adjusted for nonresponse (no adjustment of design weights). This additional measure is related to the R-indicator and depends on the variable of interest. Like the R-indicator, the maximal bias depends on the proper specification of a nonresponse model. Another limitation of the maximal bias is that it is based on an unadjusted estimator that is rarely used in practice. Schouten et al. (2011) extended the notion of an R-indicator to define partial R-indicators designed to evaluate the contribution of a single specified auxiliary variable to the representativeness of the respondents.

Peytchev et al. (2010) investigated an approach to reducing nonresponse bias through case prioritization. They suggested targeting individuals with lower estimated response probabilities. For instance, individuals could be given larger incentives or interviewers could have larger incentives for completing these cases. Their approach is basically equivalent to trying to increase the R-indicator (or achieving a more balanced sample). They also recommended using explanatory variables that are associated with the variables of interest when modelling the response probability so that the R-indicator is also indirectly associated with these variables.

Laflamme and Karaganis (2010) developed and implemented responsive collection designs for CATI surveys at Statistics Canada. Their approach fits well into the Groves and Heeringa (2006) framework. They considered four phases: a planning phase, an initial collection phase and two responsive design phases. The planning phase is conducted before data collection starts. It consists of analyzing previous data, determining strategies, and so on. The initial collection phase is used to evaluate different indicators to determine when the next phase should start. It corresponds to the first phase of the Groves and Heeringa (2006) framework. The two responsive design phases differ in the way cases are prioritized. The goal of the first responsive design phase is to improve response rates by targeting individuals with higher estimated response probabilities. This tends to increase 
the number of respondents, which is desirable. The goal of the second responsive design phase is to reduce the variability of response rates between domains of interest, which is essentially equivalent to increasing the R-indicator. This will likely reduce the variability of nonresponse weight adjustments, which is also desirable. Note that objectives of both phases are intuitively appealing but may be contradictory in terms of cases' prioritization. Laflamme and Karaganis (2010) tried to achieve a compromise between these conflicting objectives by separating data collection into two responsive design phases, each one focusing on a single objective. Our approach, described in Section 3, tries to make a compromise by using a single phase with a single objective function (quality indicator).

Lundquist and Särndal (2013) considered alternatives to the R-indicator based on the distance between the mean of respondents and the mean of the full sample for some auxiliary variables. They chose these alternative indicators to evaluate three experimental strategies using data of the Swedish Living Conditions Survey. Each strategy consists of breaking the sample into groups and, using different intervention points, declaring data collection terminated in a group if the response rate is above a certain target. The strategies differ in the number of intervention points and the selected target. These authors noted that their indicators improve as the target decreases. They concluded that data collection costs could be reduced by choosing a lower target response rate and suggested that these cost savings be used to improve other aspects of the survey design.

Schouten et al. (2013) proposed an interesting theoretical framework for adaptive survey designs focused on assigning collection strategies to sample units. It is apparently the first paper to develop some theory on this topic. The authors suggested maximizing quality for a given cost or, equivalently, minimizing cost for a given quality. The framework requires the choice of a quality indicator such as the overall response rate, the R-indicator, the maximal bias, and so on. The authors did not provide any firm recommendation regarding the choice of an appropriate indicator and a cost function. Our approach fits into this framework in the sense that we maximize quality for a given cost. Our approach is also related to the methodology in Choudhry et al. (2011), although they looked at a different problem. They investigated opportunities for improving the data collection process by focusing on interviewer allocation, whereas we focus on call prioritization.

\section{Call Prioritization Procedure}

In this Section, we develop a procedure for call prioritization in the context of CATI surveys. The reason for the restriction to CATI surveys is that it is easier to come up with a cost function since the overall cost is highly related to the total time used to conduct data collection. Our procedure aims at maximizing quality given a fixed overall budget. As pointed out in Section 1, our quality indicator is the nonresponse variance of a nonresponse-adjusted estimator.

\subsection{A Nonresponse-Adjusted Estimator and Its Nonresponse Variance}

Let $y_{i}$ be the value of a variable of interest $y$ for unit $i$ of the finite population $U$ of size $N$, and $\theta=\sum_{i \in U} y_{i}$ be the population total to be estimated. Let $s$ be a sample of size $n$ selected from $U$ through some probability sampling design $p(s)$. Let $s_{r}$ be the set of respondents of size $n_{r}$ observed at the end of data collection and generated according to 
some nonresponse mechanism, $q\left(s_{r} \mid s\right)$, which depends on the data collection procedures. Denote a nonresponse adjustment cell by the subscript $g$, for $g=1, \ldots, G$, where $G$ is the number of cells. These nonresponse adjustment cells are assumed to be known before data collection and are deemed to be homogeneous with respect to the propensity to respond to the survey. For instance, they may be some important domains of interest. Let $s_{g}$ of size $n_{g}$ be the sample units falling in cell $g$ and $s_{r g}$ of size $n_{r g}$ be the set of respondents in cell $g$ at the end of data collection. Every sample unit belongs to one and only one cell. We suppose that the estimator that would be used to estimate $\theta$ under complete response is the expansion estimator, which in our notation can be written as $\hat{\theta}=\sum_{g=1}^{G} \sum_{i \in s_{g}} w_{g i} y_{g i}$, where $y_{g i}$ is the $y$-value of unit $i$ in cell $g, w_{g i}=1 / \pi_{g i}$ is its design weight and $\pi_{g i}=$ $\operatorname{Pr}\left(i \in s_{g}\right)$ is its selection probability. The expansion estimator $\hat{\theta}$ is $p$-unbiased (design-unbiased) for $\theta$ in the sense that $E_{p}(\hat{\theta})=\theta$. The subscript $p$ indicates that the expectation is evaluated with respect to the sampling design.

Let us denote by $\rho_{g i}=\operatorname{Pr}\left(i \in s_{r g} \mid s, i \in s_{g}\right)$ the probability that sample unit $i$ in cell $g$ is a respondent to the survey at the end of data collection. It can be interpreted as the proportion of times sample unit $i$ would respond to the survey if data collection could be repeated independently an infinite number of times, always with the same procedures and the same sample $s$. Obviously, the response probability $\rho_{g i}$ depends on the data collection procedures and, more specifically, on the resources spent to obtain a response from unit $i$ in cell $g$. In the sequel, we assume uniform nonresponse within cells; that is, all sample units $i \in s$ respond independently of one another and all sample units $i \in s_{g}$ have the same probability of response to the survey; that is, $\rho_{g i} \equiv \rho_{g}$ for all $i \in s_{g}$. The response probability $\rho_{g i}$ may vary from one cell to another but is assumed to be constant within a cell. This is a standard assumption in the survey nonresponse literature. Assuming that $\rho_{g}$ is known, $g=1, \ldots, G$, a nonresponse-adjusted estimator of $\theta$ is:

$$
\tilde{\theta}_{A}=\sum_{g=1}^{G} \sum_{i \in s_{r g}} \frac{w_{g i}}{\rho_{g}} y_{g i} .
$$

It is $q$-unbiased for $\hat{\theta}$ in the sense that $E_{q}\left(\tilde{\theta}_{A} \mid s\right)=\hat{\theta}$. The subscript $q$ indicates that the expectation is evaluated with respect to the nonresponse mechanism. As a result, the adjusted estimator $\tilde{\theta}_{A}$ is also $p q$-unbiased for $\theta$; that is, $E_{p q}\left(\tilde{\theta}_{A}\right)=\theta$. In practice, the response probabilities $\rho_{g}$ are unknown and must be estimated. A possible $q$-unbiased estimator is the response rate in cell $g, \hat{\rho}_{g}=n_{r g} / n_{g}$. Since $E_{q}\left(\hat{\rho}_{g} \mid s\right)=\rho_{g}$, the response probability $\rho_{g}$ can be interpreted as the expected response rate in cell $g$. The use of $\hat{\rho}_{g}$ leads to the standard nonresponse-adjusted estimator of $\theta$ :

$$
\hat{\theta}_{A}=\sum_{g=1}^{G} \sum_{i \in s_{r g}} \frac{w_{g i}}{\hat{\rho}_{g}} y_{g i} .
$$

The estimator $\hat{\theta}_{A}$ is not $q$-unbiased for $\hat{\theta}$, unlike $\tilde{\theta}_{A}$. However, under certain conditions, including a large sample size, the squared nonresponse bias of $\hat{\theta}_{A},\left\{E_{q}\left(\hat{\theta}_{A}-\hat{\theta} \mid s\right)\right\}^{2}$, is small compared with its nonresponse variance, $\operatorname{var}_{q}\left(\hat{\theta}_{A} \mid s\right)$. We make this assumption and consider the nonresponse variance of the adjusted estimator $(1), \operatorname{var}_{q}\left(\hat{\theta}_{A} \mid s\right)$, as our quality indicator. We choose to condition on the sample $s$ to define our quality indicator because 
data collection procedures have an impact only on the nonresponse mechanism, $q\left(s_{r} \mid s\right)$, and not on the sampling design, $p(s)$. They have also no effect on the sampling error, $\hat{\theta}-\theta$, and can only reduce the nonresponse error, $\hat{\theta}_{A}-\hat{\theta}$. The nonresponse variance of the adjusted estimator (1) is approximated through a first-order Taylor linearization by

$$
\operatorname{var}_{q}\left(\hat{\theta}_{A} \mid s\right) \cong \sum_{g=1}^{G}\left(\rho_{g}^{-1}-1\right)\left(n_{g}-1\right) S_{w y, g}^{2}
$$

where

$$
S_{w y, g}^{2}=\frac{1}{n_{g}-1} \sum_{i \in s_{g}}\left(w_{g i} y_{g i}-\hat{\mu}_{g}\right)^{2} \text { and } \hat{\mu}_{g}=\frac{1}{n_{g}} \sum_{i \in s_{g}} w_{g i} y_{g i} .
$$

Remark 1: The variance $S_{w y, g}^{2}$ is variable specific and typically unknown. It could be estimated using data from a previous period of the survey (see Subsection 3.5). When there are many variables of interest, a compromise must be made. This is an issue similar to sample allocation in stratified sampling. The typical solution used in practice consists of replacing the variables of interest by a single variable $x$ that is hopefully strongly associated with the main variables of interest. Data reduction techniques such as principal component analysis could possibly be used to obtain such a variable.

Remark 2: The nonresponse-adjusted estimator (1) has a small nonresponse bias if the sample size is large and if uniform nonresponse within cells is a reasonable assumption. When the nonresponse bias is small, it makes sense to consider a data collection procedure that minimizes the nonresponse variance (2). If the uniform nonresponse assumption is not valid, then the nonresponse-adjusted estimator (1) may become substantially biased. However, this nonresponse bias cannot be eliminated at the data collection stage if no additional information on the nonrespondents is used, as evidenced in our simulation study in Section 4 (see response scenario I in Table 1). This is why we ignore the nonresponse bias and focus on the minimization of the nonresponse variance.

Remark 3: Calibration is often performed after nonresponse weight adjustment. By using linearization techniques, it would not be difficult to extend our approach to account for calibration. For simplicity, we restrict our study to the non-calibrated estimator $\hat{\theta}_{A}$ in (1).

\subsection{The Overall Cost and Its Expectation}

We assume that the overall cost of the survey depends only on $C_{N R, g}, C_{R, g}$ and $m_{g i}$, which are the cost of an unsuccessful attempt in cell $g$, the cost of an interview in cell $g$ and the total number of attempts at the end of data collection for unit $i$ in cell $g$, respectively. The overall cost can thus be expressed as:

$$
C_{T O T}=\sum_{g=1}^{G} C_{T O T, g}
$$


where

$$
C_{T O T, g}=\sum_{i \in s_{r g}}\left[\left(m_{g i}-1\right) C_{N R, g}+C_{R, g}\right]+\sum_{i \in s_{g}-s_{r g}} m_{g i} C_{N R, g}
$$

The expected overall cost is given by

$$
\tilde{C}_{T O T}=E_{q}\left(C_{T O T} \mid s\right)=\sum_{g=1}^{G} \tilde{C}_{T O T, g},
$$

where

$$
\tilde{C}_{T O T, g}=E_{q}\left(C_{T O T, g} \mid s\right)=\left(C_{R, g}-C_{N R, g}\right) n_{g} \rho_{g}+C_{N R, g} \sum_{i \in s_{g}} \tilde{m}_{g i}
$$

and $\tilde{m}_{g i}=E_{q}\left(m_{g i} \mid s\right)$ is the expected number of attempts made at the end of data collection for unit $i$ in cell $g$. Suppose the number of calls for unit $i$ in cell $g$ is restricted to be no greater than a certain fixed value, $M_{g i}$, which is known as the cap on calls for unit $i$ in cell $g$. Note that we allow the cap on calls to differ between sample units although in practice it is often set to a constant. The expected number of attempts $\tilde{m}_{g i}$ depends on the probability of response at each call attempt for unit $i$ in cell $g$, denoted by $p_{g i}$, and the cap on calls, $M_{g i}$. Note that $p_{g i}$ is different from the probability of response to the survey, $\rho_{g i}$, introduced in Subsection 3.1. The expected number of attempts $\tilde{m}_{g i}$ depends not only on $p_{g i}$ and $M_{g i}$ but also on the effort made to obtain a response for unit $i$ in cell $g$, which is itself related to the overall budget and the data collection procedures. The strict derivation of $\tilde{m}_{g i}=E_{q}\left(m_{g i} \mid s\right)$ is not straightforward. To simplify it, we make the following three assumptions:

i) The response probability $p_{g i}$ is constant from one attempt to the next.

ii) For any given sample unit, response is independent from one attempt to the next.

iii) At the end of data collection, every sample unit is either a respondent or has reached the cap on calls.

Assumption (i) implies that the response probability $p_{g i}$ does not depend on characteristics that vary over time for sample units. Assumption (ii) is more realistic if a certain amount of time is imposed between two successive calls. Assumption (iii) means that a sample unit cannot be a nonrespondent without having reached the cap on calls. It would be satisfied if the overall budget is sufficiently large (and there is no refusal). A consequence of this assumption is that the expected number of attempts $\tilde{m}_{g i}$ is only a function of $p_{g i}$ and $M_{g i}$. Although we recognize that these three assumptions may not always be satisfied in practice, we believe that they provide a useful approximation to $E_{q}\left(m_{g i} \mid s\right)$. This is confirmed in our empirical study in Section 4. Using these three assumptions, we obtain:

$$
\begin{aligned}
\tilde{m}_{g i} & =E_{q}\left(m_{g i} \mid s\right) \\
& =\left(\sum_{t=1}^{M_{g i}-1} t p_{g i}\left(1-p_{g i}\right)^{t-1}\right)+M_{g i}\left(1-p_{g i}\right)^{M_{g i}-1}=\frac{1}{p_{g i}}\left(1-\left(1-p_{g i}\right)^{M_{g i}}\right) .
\end{aligned}
$$

The algebra to go from the second to the third equation in (3) is simple but tedious and is thus omitted. 
Since $\tilde{m}_{g i}$ in (3) is only a function of $p_{g i}$ and $M_{g i}$, the expected overall cost becomes a linear function of the expected response rates, $\rho_{g}, g=1, \ldots, G$ :

$$
\tilde{C}_{T O T}=\lambda_{0}+\sum_{g=1}^{G} \lambda_{1 g} \rho_{g}
$$

with $\lambda_{0}=\sum_{g=1}^{G} C_{N R, g} \sum_{i \in s_{g}} \tilde{m}_{g i}$ and $\lambda_{1 g}=\left(C_{R, g}-C_{N R, g}\right) n_{g}$.

\subsection{The Optimization Problem and Its Solution}

Our objective consists of finding the target expected response rates, $\rho_{T g}, g=1, \ldots, G$, that minimize the nonresponse variance (2), with $\rho_{g}$ replaced by $\rho_{\mathrm{Tg}}$, subject to the budget constraint, $\lambda_{0}+\sum_{g=1}^{G} \lambda_{1 g} \rho_{T g}=K$, for a constant $K$ that represents the overall budget. The solution is:

$$
\rho_{T g}=\sqrt{\frac{\left(n_{g}-1\right) S_{w y, g}^{2}}{\delta \lambda_{1 g}}}=\sqrt{\left(\frac{n_{g}-1}{n_{g}}\right) \frac{S_{w y, g}^{2}}{\delta\left(C_{R, g}-C_{N R, g}\right)}},
$$

where

$$
\delta=\frac{\left(\sum_{g} \sqrt{\lambda_{1 g}\left(n_{g}-1\right) S_{w y, g}^{2}}\right)^{2}}{\left(K-\lambda_{0}\right)^{2}} .
$$

If $C_{R, g}$ and $C_{N R, g}$ are constant from one cell to another and if $n_{g}$ is large, then $\rho_{T g} \tilde{\propto} S_{w y, g}$, which is a solution similar to the sampling fraction obtained using Neyman allocation in stratified sampling. Moreover, if $S_{w y, g}, g=1, \ldots, G$, are found to be constant then the resulting target expected response rates $\rho_{T g}$ are also constant and thus maximize the R-indicator. However, our proposed solution does not generally maximize the R-indicator.

Unfortunately, nothing guarantees that the target expected response rates $\rho_{T g}$ are smaller than 1. If some of the $\rho_{T g}, g=1, \ldots, G$, are not smaller than 1 , they must be replaced by a value smaller but close to 1 (see Subsection 4.1 for a possible choice).

It might be useful to graph the minimum nonresponse variance, obtained using (2) with $\rho_{g}$ replaced by $\rho_{T g}$ in (5), as a function of the overall budget $K$. The minimum nonresponse variance should decrease as the budget increases. There may be a value of budget above which the minimum nonresponse variance cannot be reduced significantly and it may not be justified to spend more than that value.

\subsection{Procedure for the Selection of Cases to Be Interviewed}

Once the target expected response rates $\rho_{T g}$ have been determined, we must find the effort needed to achieve these targets. Let $e_{g i}$ be the maximum effort (in terms of the number of attempts) associated with unit $i$ in cell $g$. Under assumptions (i) and (ii) and assuming that unit $i$ in cell $g$ will be attempted at most $e_{g i}$ times, its response probability to the survey is $\rho_{g i}=1-\left(1-p_{g i}\right)^{e_{g i}}$. It is the probability that there is a response in no more than $e_{g i}$ attempts. We now want to find the effort $e_{g i}$ that makes this response probability equal to the target expected response rate $\rho_{T g}$ for each sample unit. This yields 


$$
e_{g i}=\frac{\ln \left(1-\rho_{T g}\right)}{\ln \left(1-p_{g i}\right)} .
$$

Our call procedure consists of selecting cases to be interviewed with probability proportional to the effort $e_{g i}$. The effort in (7) increases with the target expected response rate and decreases with the response probability at each attempt. A larger response probability at each attempt indicates that this unit is easier to contact and thus requires less effort to reach the target expected response rate. Note that it might sometimes be advisable to trim large values of $e_{g i}$, which may occur because of small response probabilities $p_{g i}$, so as to avoid unduly large efforts for some units. This will prevent spending a large portion of the budget for such units, especially if there is no cap on calls.

\subsection{Estimation of $S_{w y, g}^{2}$ and $p_{g i}$}

In practice, $S_{w y, g}^{2}$ and $p_{g i}$ are unknown and must be estimated. In a repeated survey, a natural choice is to use data collected at a previous point in time to obtain estimates of both $S_{w y, g}^{2}$ and $p_{g i}$. The estimation of $p_{g i}$ requires the contact history as $p_{g i}$ is the probability of response at a given attempt and not the probability of response to the survey. Paradata such as the number of call attempts should be incorporated in the response probability model if revision of the solution is considered during data collection (see Subsection 3.6). Even though the number of call attempts varies over the data collection period for each sample unit, this does not necessarily invalidate the above assumption (i). It may well be that the true response probability depends on a variable that does not vary over the data collection period but that is not observed and thus cannot be used in the model. At a certain point during data collection, some of the noninterviewed units will have been attempted many times. Such units are likely to be associated with a lower response probability $p_{g i}$. As a result, the response rate of units with a large number of attempts is expected to be smaller than the response rate of units with a small number of attempts. Therefore, a response probability model that uses the number of attempts should yield estimated response probabilities that become closer to the true response probabilities as data collection progresses than a response probability model that ignores the number of attempts. Other paradata may also be useful, such as information on the data collection mode or the time of the interview.

Estimates of $S_{w y, g}^{2}$ and $p_{g i}$ are denoted by $\hat{S}_{w y, g}^{2}$ and $\hat{p}_{g i}$, respectively. Once they are obtained, they must replace $S_{w y, g}^{2}$ and $p_{g i}$ in the solution of the optimization problem and in the selection of cases. The resulting target expected response rate and effort are denoted $\hat{\rho}_{T g}$ and $\hat{e}_{g i}$, respectively.

\subsection{Revision of the Solution to the Optimization Problem}

The solution to the above optimization problem is found before data collection starts. However, it may be desirable to revise the solution periodically (e.g., daily) as data collection progresses. Consider a revision at time $t$ whereby the call prioritization procedure is temporarily suspended to allow updating of the estimated response probabilities at an attempt, the target expected response rate and the effort. Updated values of effort are then used in the call prioritization procedure when it resumes. 
With $R$ revisions in total, the call prioritization procedure is applied $R+1$ times during the data collection process; that is, at times $t=0,1, \ldots, R$. Let $\hat{p}_{g i}^{[t]}$ denote the estimated probability of response at a given attempt at time $t$. As pointed out in Subsection 3.5, paradata may be used as explanatory variables in the response probability model so that $\hat{p}_{g i}^{[t]}$ is not necessarily constant over the data collection period. The estimated probability $\hat{p}_{g i}^{[t]}$ is used in the above optimization problem, leading to a revised target expected response rate at time $t, \hat{\rho}_{T g}^{[t]}$.

The revised target expected response rate, $\hat{\rho}_{T g}^{[t]}$, must then be updated to account for the units that have already responded. Define $n_{r g}^{[t]}$ as the number of respondents in cell $g$ at time $t$ with $n_{r g}^{[0]}=0$. The actual response rate in cell $g$ at time $t$ is: $\hat{\rho}_{g}^{[t]}=n_{r g}^{[t]} / n_{g}$. The target expected number of respondents in cell $g$ at time $t$ is $n_{g} \hat{\rho}_{T g}^{[t]}$, so that $n_{g} \hat{\rho}_{T g}^{[t]}-n_{r g}^{[t]}$ is the expected number of respondents that still remain to be interviewed. The total number of units that have not yet been interviewed at time $t$ is $n_{g}-n_{r g}^{[t]}$. We thus suggest using the updated target expected response rate,

$$
\hat{\rho}_{T g}^{*[t]}=\frac{n_{g} \hat{\rho}_{T g}^{[t]}-n_{r g}^{[t]}}{n_{g}-n_{r g}^{[t]}}=\frac{\hat{\rho}_{T g}^{[t]}-\hat{\rho}_{g}^{[t]}}{1-\hat{\rho}_{g}^{[t]}},
$$

to account for units that have already responded. Using (8), we obtain the updated effort at time $t$,

$$
\hat{e}_{g i}^{*}[t]=\frac{\ln \left(1-\hat{\rho}_{T g}^{*[t]}\right)}{\ln \left(1-\hat{p}_{g i}^{[t]}\right)} .
$$

Unsurprisingly, less effort is spent in the cells for which the response rate $\hat{\rho}_{g}^{[t]}$ is already close to the revised target expected response rate $\hat{\rho}_{T g}^{[t]}$. It is even possible that $\hat{\rho}_{g}^{[t]}>\hat{\rho}_{T g}^{[t]}$, which yields negative values of $\hat{\rho}_{T g}^{*[t]}$ and $\hat{e}_{g i}^{*}$. In such cases, the effort should be focused on the cells for which the effort $\hat{e}_{g i}^{*}[t]$ is positive.

\section{Numerical Example}

We simulated the proposed call prioritization procedure to investigate some aspects of performance and compared it with a few alternatives.

\subsection{Description of the Simulation Experiment}

We used data of the 2005 Workplace and Employee Survey (WES) conducted at Statistics Canada. Our sample, s, consists of 773 business locations in the Atlantic provinces for which a unique identifier, a design weight, stratum information used for cell assignment and gross payroll (the variable of interest $y$ ) are available. For illustration purposes, we considered three cells defined using the number of employees in a workplace. The resulting sample sizes for the three groups were $n_{1}=305, n_{2}=188$ and $n_{3}=280$.

The cost of a nonresponse attempt, the cost of an interview and the cap on calls were set to $C_{N R, g}=1, C_{R, g}=25$ and $M_{g i}=25$, respectively. Note that $C_{N R, g}$ and $C_{R, g}$ can be interpreted as time units in this experiment. Furthermore, the overall budget was set to 
$K=20,000$. The quantity $S_{w y, g}^{2}$ was estimated using a previous iteration of the survey, the 2003 WES data in the Atlantic provinces.

We considered three response scenarios that differ in the way the true probability of response at an attempt, $p_{g i}$, is defined. In each scenario, response is generated according to assumptions (i) and (ii). In addition, response is generated independently from one unit to another. The response probability $p_{g i}$ for the three scenarios is given as follows:

(C) Uniform: The probability of response is constant with $p_{g i}=0.1, i \in s$;

(G) Uniform within cells: The probability of response varies by cell with units in the same cell having the same probability (i.e., $p_{g i}=p_{g}, i \in s_{g}$ ) with $p_{1}=0.24, p_{2}=0.16$, and $p_{3}=0.04$. The average response probability over all units $i \in s$ is equal to 0.15 ;

(I) Not missing at random: The probability of response, $p_{g i}$, depends on gross payroll, $y_{g i}$, and thus varies from one unit to another. It is defined such that the average response probability over all units $i \in s$ is equal to 0.15 . The coefficient of correlation between $p_{g i}$ and $y_{g i}$ is 0.67 .

Several effort scenarios were also considered: $(C) e_{g i}=$ constant $(P)$ proposed definition in (9) $(R) \hat{e}_{g i}^{[t]} \propto-1 / \ln \left(1-\hat{p}_{g i}^{[t]}\right)(E) \hat{e}_{g i}^{[t]} \propto\left(\hat{p}_{g i}^{[t]}\right)^{10}$. The effort scenario R is obtained by using (9) with constant target expected response rates. It is thus designed to maximize the R-indicator of Schouten et al. (2009). The effort scenario E was added to see the effect of maximizing (approximately) the overall response rate.

For effort scenarios $\mathrm{P}, \mathrm{R}$, and $\mathrm{E}$, effort at the start of data collection, referred to as initial effort, and revised effort were functions of estimated response probabilities at an attempt. A response probability model was developed using the 2003 WES survey data in the Atlantic provinces. Real call data information for this previous sample was no longer available. Therefore, a single set of respondents was generated for each response scenario using a constant effort and the simulated call history was saved for each sample unit. For each response scenario, we modeled the response probability $p_{g i}$ as a function of the cell $g, g=1,2,3$, and the categorized number of attempts $a, a=1,2,3,4,5$. The categorized number of attempts was defined as follows: $a=1$ for 1 attempt; $a=2$ when the number of attempts is 2 or $3 ; a=3$ when number of attempts is 4 or $5 ; a=4$ when number of attempts is 6, 7 or 8 ; and $a=5$ when the number of attempts is greater than 8 . Using the simulated call history of this previous sample, the estimated response probability at an attempt was the response rate within cell and attempt categories; that is, it was computed as

$$
\begin{aligned}
\hat{p}_{g i} & \equiv \hat{p}(g, a) \\
& =\frac{\text { number of responses among observations in cell } g \text { with attempt category } a}{\text { number of observations in cell } g \text { with attempt category } a}
\end{aligned}
$$

Thus, there were 15 estimated response probabilities for each of the response scenarios $\mathrm{C}, \mathrm{G}$, and I. These probabilities were used to determine the effort for the 2005 WES sample. Since the number of attempts changes over the data collection period, the estimated response probability $\hat{p}_{g i}$ is modified at each revision. At the start of data collection we have $\hat{p}_{g i}^{[0]} \equiv \hat{p}(g, 1)$. There were two revisions in the data collection process: at time $t=1$ when $1 / 3$ of the total budget was exhausted and at time $t=2$ when $2 / 3$ of the total budget was exhausted. At times of revision, estimated response probabilities were revised by noting the 
cumulative number of attempts at time of revision $t$ and using this value to derive and update the categorized number of attempts at time of revision $t, a^{[t]}$, so that $\hat{p}_{g i}^{[t]} \equiv \hat{p}\left(g, a^{[t]}\right)$.

There is nothing in our theoretical framework to prevent a value of $\hat{\rho}_{T g}^{*} \geq 1$ or $\hat{\rho}_{T g}^{*}<0$ resulting in an undefined or negative effort. We addressed these problems as follows. If $\hat{\rho}_{T g}^{*[t]} \geq 1$ then the effort for the units in that cell was set to one plus the maximum effort observed among all other units in $s$. If during revision $\hat{\rho}_{T g}^{[}<0$, then the revised effort for the units in that cell was set to the minimum positive effort observed among all units in $s$.

Data collection ends when the total budget $K$ is exhausted. At this point, the estimate $\hat{\theta}_{A}$ can be computed from the realized set of respondents. We generated $B=5,000$ sets of respondents for each response-effort scenario and computed $\hat{\theta}_{A}^{(b)}, b=1,2, \ldots, B$. The complete data estimate $\hat{\theta}=\sum_{g=1}^{G} \sum_{i \in s_{g}} w_{g i} y_{g i}$ is fixed in this simulation because sampling is not repeated. Monte Carlo measures of Relative Bias (RB), Mean Squared Error (MSE) and Variance (V) of the estimator $\hat{\theta}_{A}$ were used to assess the performance of various scenarios. They are given respectively as

$$
R B=\frac{(1 / \hat{\theta}) \sum_{b}\left(\hat{\theta}_{A}^{(b)}-\hat{\theta}\right)}{B}, M S E=\frac{\sum_{b}\left(\hat{\theta}_{A}^{(b)}-\hat{\theta}\right)^{2}}{B} \text { and } V=M S E-(\hat{\theta} \times R B)^{2}
$$

Then, we computed the Relative MSE (RMSE), which is the ratio of the mean squared error for each response-effort scenario to that obtained for the same response scenario with constant effort:

$$
R M S E^{\text {resp }, e f f}=\frac{M S E^{\text {resp }, e f f}}{M S E^{\text {resp }, C}},
$$

where resp $=C, G$, or $I$, eff $=C, P, R$ or $E$, and $M S E^{r e s p, e f f}$ is the Monte Carlo mean squared error for a given response-effort scenario. Similarly, we computed the Relative Variance (RV) defined as

$$
R V^{\text {resp }, e f f}=\frac{V^{\text {resp }, e f f}}{V^{\text {resp }, C}} .
$$

The RV could be termed call prioritization effect by analogy with the notion of design effect, which is used to measure the impact on the variance of a complex sampling design compared with simple random sampling. In addition to the above measures, we computed the average over the 5,000 repetitions of the overall response rate and of the R-indicator.

\subsection{Results}

Table 1 gives summary statistics for all the response-effort scenarios considered. As expected, the proposed effort scenario was the most efficient for the three response scenarios with the smallest $R M S E^{\text {resp }, e f f}$ and $R V^{\text {resp,eff }}$. Response scenarios $\mathrm{C}$ and $\mathrm{G}$ yielded approximately unbiased estimates for all effort scenarios. The effort scenario $\mathrm{E}$ maximized the overall response rate in all cases. As demonstrated in the (resp, eff) scenario $(\mathrm{G}, \mathrm{E})$, the overall response rate is not necessarily a good indicator of the nonresponse variance or of nonresponse MSE. The effort scenario $\mathrm{R}$ is intended to maximize the R-indicator. Our results showed that there does not seem to be any relationship between the R-indicator and the nonresponse bias or variance. The response scenario I leads to large nonresponse biases that 
Table 1. Simulation statistics based on 5,000 repetitions for various response and effort scenarios

\begin{tabular}{|c|c|c|c|c|c|c|}
\hline \multicolumn{2}{|c|}{ Scenario } & \multirow[b]{2}{*}{$\begin{array}{l}R B \\
(\%)\end{array}$} & \multirow[b]{2}{*}{$\begin{array}{c}R M S E^{\text {resp,eff }} \\
(\%)\end{array}$} & \multirow[b]{2}{*}{$\begin{array}{c}R V^{\text {resp,eff }} \\
(\%)\end{array}$} & \multirow{2}{*}{$\begin{array}{l}\text { Average } \\
\text { overall } \\
\text { response } \\
\text { rate }(\%)\end{array}$} & \multirow[b]{2}{*}{$\begin{array}{l}\text { Average } \\
\text { R-indicator } \\
\quad(\%)\end{array}$} \\
\hline $\begin{array}{l}\text { Response } \\
\text { Scenario }\end{array}$ & $\begin{array}{c}\text { Effort } \\
\text { Scenario }\end{array}$ & & & & & \\
\hline $\mathrm{C}$ & $\mathrm{P}$ & -0.118 & 61.1 & 61.0 & 76.1 & 87.6 \\
\hline $\mathrm{C}$ & $\mathrm{C}$ & 0.044 & 100.0 & 100.0 & 76.1 & 97.3 \\
\hline $\mathrm{C}$ & $\mathrm{R}$ & 0.024 & 95.2 & 95.2 & 76.1 & 96.1 \\
\hline $\mathrm{C}$ & $\mathrm{E}$ & 0.034 & 133.0 & 133.0 & 76.1 & 77.2 \\
\hline $\mathrm{G}$ & $\mathrm{P}$ & -0.158 & 53.9 & 53.8 & 73.7 & 75.7 \\
\hline $\mathrm{G}$ & $\mathrm{C}$ & 0.123 & 100.0 & 100.0 & 78.3 & 64.2 \\
\hline $\mathrm{G}$ & $\mathrm{R}$ & -0.196 & 66.2 & 66.1 & 74.0 & 86.9 \\
\hline $\mathrm{G}$ & $\mathrm{E}$ & 0.203 & 111.7 & 111.7 & 79.2 & 56.6 \\
\hline I & $\mathrm{P}$ & 11.013 & 91.8 & 70.2 & 77.2 & 87.2 \\
\hline I & $\mathrm{C}$ & 11.406 & 100.0 & 100.0 & 79.4 & 82.1 \\
\hline I & $\mathrm{R}$ & 11.863 & 106.0 & 73.4 & 78.0 & 95.3 \\
\hline I & $\mathrm{E}$ & 10.506 & 92.0 & 197.1 & 80.0 & 67.6 \\
\hline
\end{tabular}

are similar for all the effort scenarios. This is because missing values are not missing at random and the true response probabilities $p_{g i}$ are not estimated accurately. As expected, none of the effort scenarios can completely eliminate the nonresponse bias in this case. For each of the response scenarios, the different effort scenarios led to similar nonresponse biases. In other words, the use of cells at the data collection stage did not have any significant effect on the nonresponse bias because this information was already used at the estimation stage. It is thus not possible to favor one effort scenario based solely on nonresponse bias. Based on nonresponse variance and MSE, our approach was the best in this simulation study.

Out of curiosity, we ran the response scenario I using the known probabilities of response $p_{g i}$ in the calculation of efforts instead of the estimated response probabilities. This case never occurs in practice but is informative to better understand the reasons for the nonresponse biases in the response scenario I in Table 1. These results are shown in Table 2. The nonresponse bias is reduced significantly for the effort scenario $\mathrm{R}$ because this scenario can take advantage of the additional information available when $p_{g i}$ is known. Note that our proposed approach also succeeded in reducing the nonresponse bias even though it is focused on reducing the nonresponse variance. Note also that the information contained in $p_{g i}$ (when it is known) could be used to improve the estimator $\hat{\theta}_{A}$; for example, by making nonresponse classes homogeneous with respect to $p_{g i}$. This would result in a reduced nonresponse bias for all the effort scenarios because we would be in a situation similar to the response scenario $\mathrm{G}$ in Table 1 .

\section{Conclusion}

We have proposed a call prioritization procedure that attempts to minimize the nonresponse variance of a nonresponse-adjusted estimator subject to an overall budget constraint. Our empirical study showed that it had a smaller mean squared error than alternatives such as maximizing the R-indicator for three different nonresponse mechanisms.

Although we focused on the reduction of the nonresponse variance, we believe that the reduction of the nonresponse bias remains a more important issue in practice. However, 
Table 2. Simulation statistics based on 5,000 repetitions for the response scenario I and various effort scenarios using known probabilities of response at an attempt

\begin{tabular}{|c|c|c|c|c|c|c|}
\hline \multicolumn{2}{|c|}{ Scenario } & \multirow[b]{2}{*}{$\begin{array}{l}R B \\
(\%)\end{array}$} & \multirow[b]{2}{*}{$\begin{array}{c}R M S E^{r e s p, e f f} \\
(\%)\end{array}$} & \multirow[b]{2}{*}{$\begin{array}{c}R V^{\text {resp,eff }} \\
(\%)\end{array}$} & \multirow[b]{2}{*}{$\begin{array}{l}\text { Average } \\
\text { overall } \\
\text { response } \\
\text { rate }(\%)\end{array}$} & \multirow[b]{2}{*}{$\begin{array}{c}\text { Average } \\
\text { R-indicator } \\
(\%)\end{array}$} \\
\hline $\begin{array}{l}\text { Response } \\
\text { Scenario }\end{array}$ & $\begin{array}{c}\text { Effort } \\
\text { Scenario } \\
\text { (using known } \\
\text { probabilities } \\
\text { of response) }\end{array}$ & & & & & \\
\hline I & $\mathrm{P}$ & 1.862 & 9.7 & 113.5 & 76.4 & 86.6 \\
\hline I & $\mathrm{C}$ & 11.406 & 100.0 & 100.0 & 79.4 & 82.1 \\
\hline I & $\mathrm{R}$ & -0.636 & 14.8 & 217.2 & 76.7 & 97.2 \\
\hline I & $\mathrm{E}$ & 18.224 & 241.5 & 38.8 & 81.0 & 71.0 \\
\hline
\end{tabular}

we think that a call prioritization procedure cannot reduce the nonresponse bias to a better extent than a proper nonresponse weight adjustment. This is confirmed in our empirical study, since all the data collection procedures tested led to similar nonresponse biases. Hansen and Hurwitz (1946) proposed an approach to reducing the nonresponse bias at the data collection stage of a survey by randomly selecting a subsample of nonrespondents after a certain point during data collection. Indeed, their approach completely eliminates the nonresponse bias if all the units selected in the subsample respond. The latter condition is not realistic in practice and nonresponse is usually present in the subsample, which is likely to result in nonresponse bias. We conjecture that the method of Hansen and Hurwitz (1946) can achieve some nonresponse bias reduction even if there is some nonresponse in the subsample of nonrespondents. If the method is used, our call prioritization procedure can still be applied within the subsample to reduce the nonresponse variance.

Our procedure could be improved in a number of ways. For instance, we could distinguish two different types of nonrespondents at the end of data collection: those who have refused to respond to the survey and those who have not been contacted. The probability that a given unit $i \in s_{g}$ is a respondent to the survey at the end of data collection, $\rho_{g}$, would then become the product of two different probabilities: the probability that unit $i$ is contacted at the end of data collection, denoted by $\rho_{g}^{c}$, and the probability that unit $i$ responds to the survey once contacted, denoted by $\rho_{g}^{r \mid c}$. A call prioritization procedure only has an effect on the contact probability $\rho_{g}^{c}$. Similarly to Subsection 3.3, the goal would be to find the target contact probabilities that minimize the nonresponse variance subject to a budget constraint. The cost function would need to be modified to account for the difference between the cost of a refusal and the cost of a noncontact. Also, no further call attempt is made after a refusal, unlike a noncontact. The probability of response at a given call attempt for a unit $i \in s_{g}, p_{g i}$, would be modeled in two steps similarly to the probability $\rho_{g}$, as described above. Although this idea was not fully developed in our article and still requires further thought, we believe that it would not be too difficult to extend our approach so as to handle different types of nonrespondents.

Further research and improvements remain to be done to make the approach even more useful. Our notion of effort was defined in terms of number of call attempts. It might be possible to extend our definition of effort so as to cover other types of data collection features such as incentives. This definitely requires further thought. Another improvement 
would be to restrict the estimated target expected response rate at time $t, \hat{\rho}_{T g}^{[t]}$, to be no smaller than the achieved response rate at time $t, \hat{\rho}_{g}^{[t]}$, but smaller than 1 ; that is, $\hat{\rho}_{g}^{[t]} \leq \hat{\rho}_{T g}^{[t]}<1$. This inequality constraint would require an iterative algorithm since a closed-form solution to the optimization problem would no longer be possible. Finally, it would be useful to relax the three assumptions made to obtain the expected number of attempts $\tilde{m}_{g i}$. In particular, it should be investigated how to relax assumption (iii) so as to account for the fact that the budget is not unlimited and that the effort $e_{g i}$ has an effect on $\tilde{m}_{g i}$.

\section{References}

Axinn, W.G., C.F. Link, and R.M. Groves. 2011. "Responsive Survey Design, Demographic Data Collection, and Models of Demographic Behavior." Demography 48: 1127-1149. DOI: http://dx.doi.org/10.1007/s13524-011-0044-1.

Choudhry, G.H., M.A. Hidiroglou, and F. Laflamme. 2011. Optimizing CATI Workload Simultaneously Minimize Data Collection Cost for Several Surveys, Technical Report Presented at Statistics Canada's Advisory Committee on Statistical Methods, October 31-November 1, 2011.

Groves, R.M. and S.G. Heeringa. 2006. "Responsive Design for Household Surveys: Tools for Actively Controlling Survey Errors and Costs." Journal of the Royal Statistical Society, Series A 169: 439-457. DOI: http://dx.doi.org/10.1111/j.1467985x.2006.00423.x.

Hansen, M.H. and W.N. Hurwitz. 1946. "The Problem of Non-response in Sample Surveys." Journal of the American Statistical Association 41: 517-529. DOI: http://dx.doi.org/10.1080/01621459.1946.10501894.

Laflamme, F. and M. Karaganis. 2010. "Implementation of Responsive Collection Design for CATI Surveys at Statistics Canada." In Proceedings of the European Conference on Quality in Official Statistics, Helsinki, Finland, May 2010. Available at: http://q2010. stat.fi./media//presentations/1_Responsive_design_paper_london_event1_revised.doc (accessed October, 2014).

Lundquist, P. and C.-E. Särndal. 2013. "Aspects of Responsive Design with Applications to the Swedish Living Conditions Survey." Journal of Official Statistics 29: 557-582. DOI: http://dx.doi.org/10.2478/jos-2013-0040.

Peytchev, A., S. Riley, J. Rosen, J. Murphy, and M. Lindblad. 2010. "Reduction of Nonresponse Bias in Surveys through Case Prioritization." Survey Research Methods 4: $21-29$.

Schouten, B., M. Calinescu, and A. Luiten. 2013. “Optimizing Quality of Response through Adaptive Survey Designs.” Survey Methodology 39: 29-58.

Schouten, B., F. Cobben, and J. Bethlehem. 2009. "Indicators for the representativeness of survey response." Survey Methodology 35: 101-113.

Schouten, B., N. Shlomo, and C. Skinner. 2011. "Indicators for Monitoring and Improving Representativeness of Response.” Journal of Official Statistics 27: 1-24.

Received January 2013

Revised February 2014

Accepted July 2014 